\title{
Nordiques
}

33 | 2017

La transition des villes nordiques : quelles innovations territoriales en périphérie?

\section{La naturbanité, une utopie réalisée par le Parc national urbain de Stockholm?}

\section{Camille Girault}

\section{(2) OpenEdition}

\section{Journals}

Édition électronique

URL : https://journals.openedition.org/nordiques/3128

DOI : $10.4000 /$ nordiques. 3128

ISSN : 2777-8479

Éditeur :

Association Norden, Bibliothèque de Caen la mer

Édition imprimée

Date de publication : 1 mai 2017

Pagination : $61-78$

ISBN : 978-2-9544654-9-4

ISSN : $1761-7677$

Référence électronique

Camille Girault, "La naturbanité, une utopie réalisée par le Parc national urbain de Stockholm ?»,

Nordiques [En ligne], 33 | 2017, mis en ligne le 13 janvier 2022, consulté le 02 février 2022. URL : http:// journals.openedition.org/nordiques/3128 ; DOI : https://doi.org/10.4000/nordiques.3128 


\section{La naturbanité, une utopie réalisée par le Parc national urbain de Stockholm ?}

Camille Girault*

\section{RÉSUMÉ}

Au prisme du Parc national urbain de Stockholm, un renversement des logiques de protection est ici envisagé comme figure de l'utopie : l'urbain n'est pas appréhendé comme une menace vis-à-vis de la nature, l'urbanité est au contraire considérée comme l'objet même de la protection. En se revendiquant comme la première ville du monde à avoir établi un tel "Parc national urbain", Stockholm nourrit l'image d'une cité idéale par l'établissement d'un espace urbain innovant. Ce dernier participe à l'identité urbaine et contribue à la production d'un rapport nordique au monde où des valeurs a priori contradictoires sont condensées en un même lieu. L'imbrication forte entre l'urbanité et la naturalité qui en résulte invite ainsi à proposer et à discuter la notion de naturbanité comme le fondement et l'expression d'une utopie réalisée dans la métropole suédoise.

\section{ABSTRACT}

Through the Stockholm National Urban Park, a reversal of the conservation logic is here considered as a figure of utopia: urban features are not a threat to nature, but urbanity is regarded as the very object worth conserving. By claiming itself as the first city in the world to have established such a "national urban park", Stockholm nurtures the image of an ideal city by creating an innovative urban space. The latter participates in urban identity and it contributes to built a Nordic relation to the world where some a priori contradictory values are condensed into the same place. The result of a strong imbrication between urbanity and naturalness invites us to propose and discuss the concept of naturbanity as the foundation and expression of an utopia carried out in the Swedish metropolis.

* Agrégé et doctorant en géographie au laboratoire Edytem (UMR 5204 - CNRS - Université Savoie Mont-Blanc), Camille Girault prépare une thèse sur les politiques de protection des espaces naturels dans les métropoles nordiques (http://edytem.univ-savoie.fr/annuaire/girault-camille). 


\section{INTRODUCTION}

Durant l'été 2016, le sculpteur Tony Cragg expose en plein air quelques-unes de ses œuvres monumentales à Djurgården, une des quatorze îles de l'archipel de Stockholm qui a donné son nom à l'un des principaux espaces verts de la capitale suédoise. Les modelés des sculptures renvoient à des formes d'un autre temps, qui auraient pu être façonnées il y a plusieurs millions d'années, alors que la pelouse bien rase sur laquelle elles se trouvent rappelle à quel point ce parc urbain est de toutes pièces une création humaine. Le promeneur est ainsi confronté à ses propres représentations; la distinction dualiste entre le naturel et l'artificiel tombe ; l'urbanité et la naturalité se confondent (photographie 1a).

Une telle réflexion aurait pu être menée dans un autre jardin public. Pourtant, elle prend ici un sens singulier en raison du statut de "Parc national urbain » attribué à cet espace. Cette caractérisation législative et juridique entraîne une réglementation spécifique, elle labellise un espace vert urbain en empruntant au vocable des politiques de protection de la nature et permet à Stockholm de revendiquer la primauté de cette initiative à l'échelle mondiale. Entre espace protégé, parc urbain et objet politique imaginé, ce Parc national urbain soulève plusieurs ambiguïtés aux yeux du géographe. En observant les différentes réalités qu'il recouvre, tant matérielles qu'idéelles, plusieurs pistes de réflexion peuvent être suivies pour comprendre au mieux le sens social, politique et symbolique de cet objet spatial.

Les lignes qui suivent proposent d'appréhender la naturalité nordique comme une construction culturelle et politique qui s'appuie en partie sur des dispositifs de protection environnementale pour répondre à une demande sociale et éminemment urbaine de contact avec le monde biophysique. Autrement dit, l'hypothèse majeure est de considérer le Parc national urbain de Stockholm comme une innovation politique qui renverse les logiques habituelles de protection. L'urbain ne serait pas une menace justifiant l'instauration d'espaces protégés ; l'urbanité est au contraire l'objet même de mesures protectrices où la dimension écologique n'est qu'un enjeu parmi d'autres. Une telle démarche fait écho à des conceptions en partie inédites des rapports entre l'homme et la nature ${ }^{1}$ : la ville n'est pas le domaine exclusif de l'artificiel, renvoyant ipso facto les espaces naturels et leur protection à son extérieur, ou au minimum à sa périphérie. Le Parc national urbain de Stockholm est, au contraire, une centralité urbaine majeure et il constitue un fait métropolitain à part entière.

1 Il est évidemment impossible de considérer la nature en dehors de l'existence humaine et de l'influence anthropique. Dire la nature, c'est toujours faire référence à des productions culturelles, à des représentations sociales et à des catégorisations politiques dominantes. Quant à l'usage de la majuscule qui s'observe parfois pour insister sur le caractère conceptuel de ce terme, nous n'y souscrivons pas ici. En effet, la distinction qui se joue à propos de nature relève moins d'une différence entre une acception commune et une acception philosophique que d'une polysémie, à la fois dans le sens profane et dans le concept scientifique. 


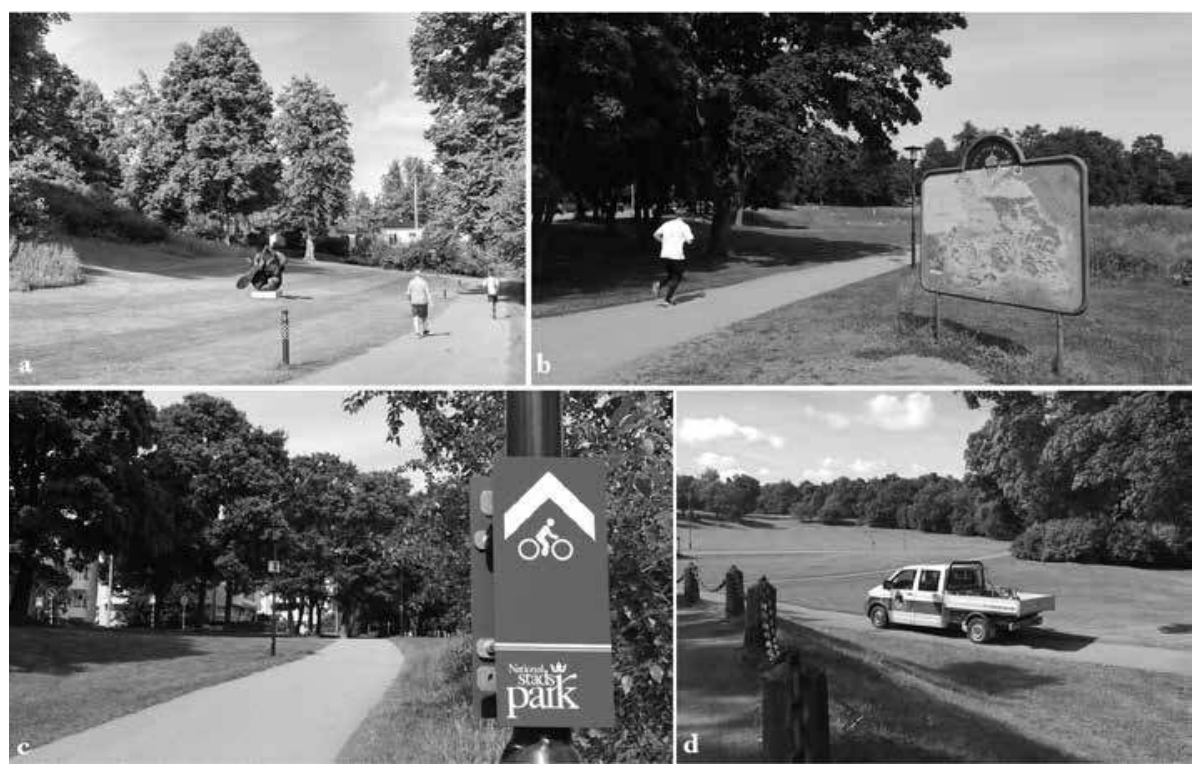

Photographies 1 : Un parc urbain à l'instar d'autres espaces verts ?

La notion d'utopie est alors des plus utiles pour penser cet espace urbain à la fois ancien et innovant, et qui traduirait un équilibre idéal entre la société et un ensemble de réalités biophysiques. S'il est inscrit dans la loi, s'il est largement pratiqué et investi, ce Parc national urbain revêt malgré tout un certain déficit d'appropriation en tant qu'objet de protection. Il ne serait donc que partiellement réalisé et pour partie irréalisable, nourrissant ainsi son caractère hybride de sa double dimension réelle et chimérique. À l'aune d'une relecture des valeurs et des finalités assignées en général aux espaces protégés, l'idée d'une naturbanité émerge et fait ici figure d'utopie. Elle condense en un même terme l'intrication de la naturalité dans l'urbanité, et réciproquement. Les approches dialectiques classiques entre la ville et la nature, entre le naturel et l'artificiel, entre l'aménagement et le ménagement se déplacent ainsi vers une tension entre espace de la quotidienneté et espace imaginé, entre pragmatisme et idéalisme, voire entre idéologie et utopie.

À l'instar de l'objet étudié, la réflexion proposée est à la croisée de la réalité observée et du construit idéel, du factuel et du théorique. Une revue de la littérature, des observations conduites in situ, des éclairages apportés par des entretiens auprès de différents acteurs, des analyses de documents législatifs, d'urbanisme et de supports de communication institutionnelle ou associative se complètent et s'articulent pour donner corps à ce propos. Cette analyse est une interprétation dépendante du regard de l'observateur et non un reflet juste, vrai et exhaustif d'un objet d'étude. 
Parcourir Djurgården, c'est d'abord constater que ce Parc national urbain relève d'une forme de protection singulière. Dans cet objet spatial hybride convergent urbanité et naturalité au sein d'un même lieu ; la naturbanité est une manière de nommer cette imbrication qui apparaît comme la figure de l'utopie.

\section{LES RESSORTS D'UNE PROTECTION SINGULIÈRE ET UTOPIQUE : FAUT-IL CHOISIR ENTRE PARC URBAIN ET ESPACE PROTÉGÉ ?}

Le Parc national urbain est une forme de protection innovante renvoyant à l'utopie à la fois par le récit urbain qu'il convoque et par le projet urbanistique qu'il incarne. Sans être véritablement un espace protégé reconnu comme tel par certains acteurs, il correspond malgré tout à un périmètre clairement établi où des mesures de protection permettent la préservation et la gestion de valeurs assignées à cet espace. Il diffère également des espaces verts plus classiques par la reconnaissance de son intérêt national et par sa dimension symbolique. L'instauration de ce Parc national urbain renforce le ménagement de l'urbanisation et participe à l'affirmation de l'urbanité de Stockholm.

\section{Le Parc national urbain, un objet spatial hybride et innovant}

Le Parc national urbain de Stockholm est fréquemment présenté, voire revendiqué, comme le premier du genre à l'échelle mondiale ${ }^{2}$. Le contexte métropolitain dans lequel il s'inscrit est une première particularité, alors que la majorité des parcs nationaux de Suède, d'Europe et du monde sont localisés à l'extérieur des zones urbanisées ${ }^{3}$. Pour autant, il n'est pas le seul ni le premier historiquement à être intégré au tissu urbain. D’autres espaces protégés urbains sont recensés dans le monde et prennent des statuts différents ${ }^{4}$.

En fait, la primauté de la capitale suédoise concernant l'objet « Parc national urbain » apparaît davantage par l'innovation statutaire, par la contraction sémantique et par l'invention urbanistique qu'il incarne que par le glissement de politiques de protection préexistantes vers un contexte métropolitain. En effet, le Parc national urbain de Stockholm ne s'appuie pas sur les mêmes lois environnementales

2 Peter Schantz, "The Formation of National Urban Parks : A Nordic Contribution to Sustainable Development ", in The European City and Green Space. London, Stockholm, Helsinki and St Petersburg, 1850-2000, P. Clark (dir.), Aldershot, Ashgate, 2006, p. 159-174 ; Ylva Uggla, "Protecting Urban Greenery : The Case of Stockholm's National City Park ", City \& Community, 13, 4, 2014a, p. 360-380.

3 Samuel Depraz, Géographie des espaces naturels protégés. Genèse, principes et enjeux territoriaux, Paris, A. Colin, 2008 ; Lionel Laslaz (dir.), Atlas mondial des espaces protégés. Les sociétés face à la nature, Paris, Autrement, 2012.

4 Emmanuel Lézy, Louise Bruno (dir.), BiodiverCités. Les aires protégées urbaines, des laboratoires grandeur nature, Paris, Éditions Le Manuscrit, 2013 ; Ted Trzyna (dir.), «Urban Protected Areas : Profiles and Best Practice Guidelines ", Best Practice Protected Area Guidelines Series, nº 22, 2014. 
que celles qui ont permis de créer les parcs nationaux du pays ${ }^{5}$. Alors que ces derniers sont nécessairement des territoires appartenant à l'État où le contrôle des différents usages et aménagements est très strict, le Parc national urbain de Stockholm fut créé en 1995 par un décret spécifique du Parlement suédois. Une création confirmée par un paragraphe dédié du Code de l'environnement de 1999.

Ainsi, l'espace constituant le Parc national urbain n'est pas obligatoirement propriété de l'État, la réglementation des activités n'est pas la même qu'au sein des parcs nationaux :

Les nouveaux aménagements, les nouveaux bâtiments et les autres changements ne sont autorisés dans les parcs nationaux urbains que s'ils peuvent être réalisés sans abîmer les paysages ou l'environnement naturel des parcs et sans porter préjudice aux autres biens naturels et culturels. ${ }^{6}$

L'organisme gestionnaire n'est pas identique non plus. Contrairement aux parcs nationaux qui sont gérés par l'Agence suédoise de protection environnementale, le Parc national urbain de Stockholm est géré par l'Administration royale de Djurgården (photographies 1).

En dépit d'un périmètre clairement établi et d'une réglementation spécifique en faveur de la préservation et de la valorisation d'un espace présentant une réelle richesse écosystémique et des valeurs patrimoniales ${ }^{7}$, le Parc national urbain de Stockholm n'est pas reconnu comme un espace protégé stricto sensu par la principale ONG environnementale agissant à l'échelle mondiale pour évaluer et promouvoir les politiques de protection de la nature. En effet, l'UICN (Union internationale pour la conservation de la nature) propose une définition de l'espace protégé ${ }^{8}$ dans laquelle la caractérisation du Parc national urbain se retrouve pleinement. Pour autant, ce dernier n'est pas recensé comme tel. S’il ne correspondait effectivement pas à la catégorie II consacrée aux parcs nationaux, il pourrait néanmoins trouver sa place au sein de la catégorie IV désignant les aires de gestion des

5 Dès 1909 , la Suède fut le premier pays européen à créer des parcs nationaux sur son territoire montrant ainsi dès le début du $\mathrm{XX}^{e}$ siècle son rôle de précurseur dans la mise en œuvre des politiques environnementales.

6 The Swedish Environmental Code, 1999, partie I, chap. 4, section 7. C'est nous qui traduisons.

7 Johan Colding, Thomas Elmqvist, Jakob Lundberg et al., The Stockholm Urban Assessment (SUASweden), 2003 ; Stephan Barthel, Johan Colding, Thomas Elmqvist, Carl Folke, " History and Local Management of a Biodiversity-rich, Urban Cultural Landscape ", Ecology and Society, 10, 2, 2005, [http://www.ecologyandsociety.org/vol10/iss2/art10/] (consulté le 27 février 2017) ; Henrik Ernstson, Stephan Barthel, Erik Andersson, Sara Borgström, "Scale-Crossing Brokers and Network Governance of Urban Ecosystem Services: The Case of Stockholm”, Ecology and Society, 15, 4, 2010, [http://www. ecologyandsociety.org/vol15/iss4/art28/] (consulté le 27 février 2017).

8 "Un espace géographique clairement défini, reconnu, consacré et géré, par tout moyen efficace, juridique ou autre, pour assurer à long terme la conservation de la nature ainsi que les services écosystémiques et les valeurs culturelles qui lui sont associés ", Nigel Dudley (éd.), Lignes directrices pour l'application des catégories de gestion aux aires protégées, Gland, UICN, 2008, p. 10. 
habitats ou des espèces qui ont entre autres pour objectif $d$ ' " offrir un moyen qui permet aux résidents des villes d'être régulièrement en contact avec la nature " L'absence de reconnaissance du Parc national urbain à l'échelle internationale par l'UICN ne saurait toutefois traduire la faiblesse de la protection. Elle exprime plutôt le caractère novateur de cet objet spatial nouvellement venu dans le vaste panel des espaces protégés. Le choix politique qui a prévalu a donc été de composer entre les différentes valeurs, attributions et finalités d'un parc urbain et d'un parc national, plutôt que d'adopter une catégorie et un dispositif déjà existants.

\section{Dire une protection différente par un renversement de la logique conservatrice}

La ville et l'architecture constituent un des thèmes privilégiés de l'utopie en tant qu'espace de projection et de production d'une société plus juste où le bienêtre des habitants est une des raisons d'être de l'action politique ${ }^{10}$. Par ailleurs, la protection de la nature est par essence politique à travers les choix qu' elle suppose et la réglementation qu'elle instaure ${ }^{11}$. Le Parc national urbain de Stockholm est ainsi un objet spatial doublement politique par son caractère urbanistique et par ce qu'il exprime de la relation de l'homme à son environnement. Les ressorts de cette protection singulière sont ainsi à chercher dans le renversement de la logique conservatrice qui est à l'œuvre avec ce parc. En effet, le processus de protection est ici socialement justifié par l'intérêt national que représente cet espace de naturalité dans le tissu urbain de la métropole suédoise. Contrairement aux parcs nationaux dont l'objectif est d'abord de protéger des écosystèmes et, ensuite, de permettre le déploiement de pratiques récréatives, le Parc national urbain de Stockholm envisage avant tout de garantir une proximité et une accessibilité des usagers à un vaste espace patrimonialisé et ménagé. Ainsi, dans les 27 kilomètres carrés de ce parc (répartis sur les communes de Lidingö, Solna et Stockholm) s'entremêlent les aspects culturels et naturels, les dimensions historiques et prospectives et les échelles locales et nationales (carte 1).

Au-delà de la vaste emprise spatiale du Parc national urbain au cœur de l'aire métropolitaine, ce sont surtout les 15 millions de visiteurs annuels ${ }^{12}$ qui en font un espace singulier au sein d'une métropole comptant 2,2 millions d'habitants et accueillant de plus en plus de touristes (plus de 4 millions par an ces dernières années). Autrement dit, le caractère pleinement urbain de cet espace ainsi que

9 Ibid., p. 23.

10 Françoise Choay, Lurbanisme, utopies et réalité. Une anthologie, Paris, Seuil, 1965 ; Thierry Paquot, Utopies et utopistes, Paris, La Découverte, 2007.

11 Yrjö Haila, "Genealogy of Nature Conservation : A Political Perspective », Nature Conservation, 1, 2012, p. 27-52 ; Catherine Larrère, Raphaël Larrère, Penser et agir avec la nature. Une enquête philosophique, Paris, La Découverte, 2015 ; Ismael Vaccaro, Oriol Beltran, Pierre-Alexandre Paquet, « Political Ecology and Conservation Policies: Some Theoretical Genealogies ", Journal of Political Ecology, 20, 2013, p. 255-272.

12 Lars Nyberg, The Royal National City Park, Stockholm, Länsstyrelsen Stockholm, 2015. 


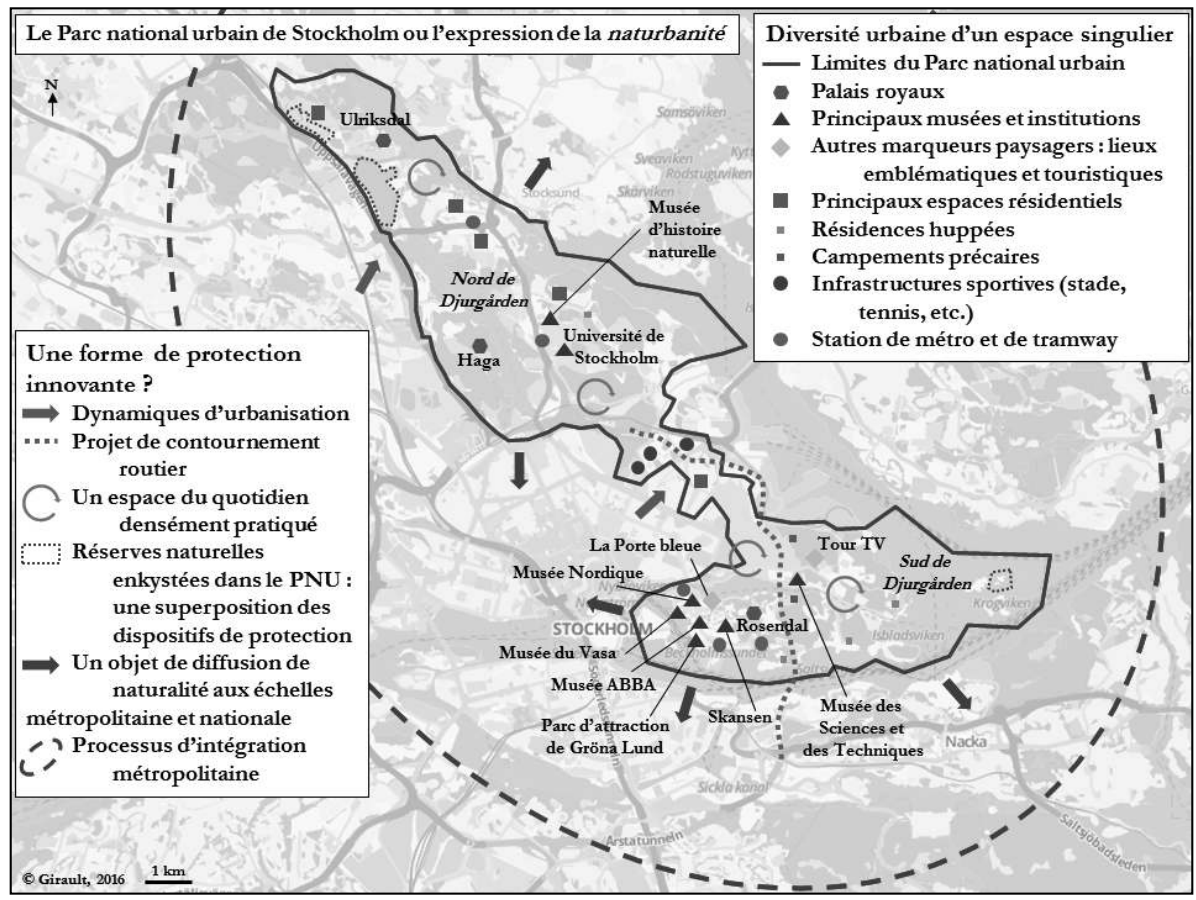

Carte 1 : Le Parc national urbain de Stockholm ou l'expression de la naturbanité?

Réalisation Girault, 2016 ; fond de carte OpenStreetMap, 2016

sa forte attractivité métropolitaine ne représentent pas des menaces vis-à-vis de la nature qui justifieraient des mesures de protection et de contrôle spatial ; au contraire, le but de la protection qui s'affirme est de maintenir ce paysage emblématique de la capitale suédoise et de consolider cette forme d'urbanité singulière où la naturalité est très prégnante, pour que les visiteurs puissent en jouir pleinement.

À ce propos, le lobbying mené par l'organisation WWF Suède pour instaurer le Parc national urbain est également une forme d'innovation puisqu'il ne s'agissait pas d'exercer des pressions politiques pour étendre un dispositif de protection existant, mais bel et bien de revendiquer la nécessité d'un statut hybride pour faire de la préservation de la spécificité de Djurgården une des dimensions centrales du mode de vie de Stockholm, donc de l'urbanité de la métropole suédoise et, plus largement, de l'urbanité des sociétés nordiques. En outre, le Parc national urbain de Stockholm est le résultat d'un processus politique présenté comme innovant et qui nourrit le paradigme de la durabilité urbaine ${ }^{13}$. Il montre que les logiques de protection environnementale ne sont pas contradictoires

13 Peter Schantz, op. cit. 
avec le fait urbain, de la même manière que d'autres projets de dépassement de l'opposition ville / nature ont pu être envisagés par l'invention de nouveaux types d'espaces protégés. Le projet inabouti et avorté de Parc naturel urbain en Suisse, qui demeure aujourd'hui à l'état d'utopie, est un autre exemple qui a été étudié récemment ${ }^{14}$. Dans le cas de Stockholm, le projet d'une protection à fronts renversés a bel et bien vu le jour. Pour autant, les acteurs écologistes considèrent aujourd'hui que la protection instaurée par le Parc national urbain reste insuffisante. Les membres du WWF Suède et de l'association Ekoparken en faveur de la préservation de Djurgården estiment qu'il faudrait étendre les réserves naturelles actuelles comprises dans le périmètre du parc (carte 1) ou en créer de nouvelles à défaut d'un renforcement de la réglementation environnementale et patrimoniale actuelle du Parc national urbain.

L'utopie réside dès lors dans l'invention d'un objet juridique nouveau dont l'objectif est de répondre au mieux aux enjeux métropolitains, au sein desquels l'imbrication actorielle et scalaire est particulièrement forte. La gouvernance mise en ouvre n'est ni celle d'un espace vert urbain traditionnel ni celle d'un parc national, mais plutôt une gestion hybride, collective et concertée.

\section{Affirmer l'urbanité d'un espace par le ménagement de son urbanisation}

En empruntant à la pensée foucaldienne, le Parc national urbain de Stockholm correspond particulièrement bien à une hétérotopi $\mathrm{e}^{15}$, cet espace autre matérialisé, réalisé et localisé qui traduit en miroir un projet utopique, en l'occurrence un contact maximal entre l'urbanité et la naturalité d'un lieu. Par sa juxtaposition spatiale, son articulation entre le réel et l'imaginaire et sa mise en relation du proche et du lointain, l'hétérotopie est à la fois une rupture forte avec l'espace environnant et un lien majeur avec le reste du monde et de la cité. Le philosophe estime que le jardin est une des meilleures illustrations du concept d'hétérotopie en raison de sa capacité à condenser en un même lieu la diversité et la densité du monde ${ }^{16}$. À de nombreux égards, cela est le cas à Stockholm au sein du parc de Djurgården. En effet, ce dernier comporte plusieurs musées et institutions emblématiques de la Suède et de la nordicité qui en font un miroir de la société. Ainsi, depuis son établissement à la fin du XIX ${ }^{e}$ siècle, le musée de plein air Skansen a pour objectif de montrer la diversité de la culture dans une perspective ethnographique. Il se veut un condensé de la société suédoise d'autrefois en permettant littéralement aux visiteurs de se promener à travers les différents siècles de

14 Gérald Hess, Joëlle Salomon Cavin, «Le Parc naturel urbain en Suisse : une utopie ? Approche philosophique pour dépasser l'opposition ville / nature ", VertigO. La revue électronique en sciences de l'environnement, 15, 1, 2015, [http://vertigo.revues.org/15874] (consulté le 27 février 2017).

15 Michel Foucault, " Des espaces autres », in Dits et Écrits II (1976-1988), Paris, Gallimard, 2001 [1984], p. 1571-1581.

16 Ibid. 


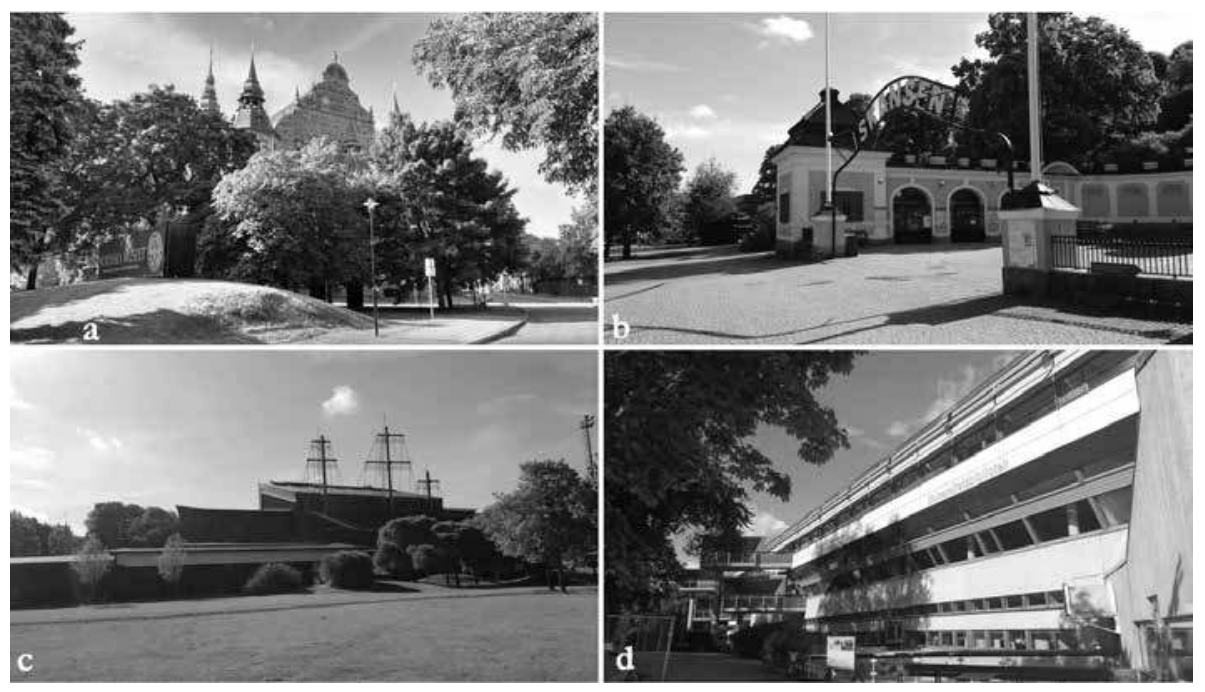

Photographies 2 : Musées et université : des institutions emblématiques au cœur du Parc national urbain, des marqueurs paysagers métropolitains. Girault, juillet 2016

l'histoire du pays, du nord au sud de la Suède. Le parc d'attraction Gröna Lund créé à la même époque est aujourd'hui l'expression d'une société des loisirs et du bien-être et offre un complément techniciste et consumériste aux aménités environnementales des espaces ouverts et végétalisés de Djurgården. Le musée Vasa, le musée nordique, celui d'ABBA, celui des Sciences et des techniques ou encore l'université de Stockholm (photographies 2) plus au nord de l'île sont d'autres exemples qui donnent corps à cette hétérotopie et qui expriment l'urbanité de Djurgården. Aussi, cette dernière est bel et bien au centre des mesures législatives et réglementaires instaurées par le Parc national urbain qui cherchent à encadrer sans restreindre ces activités culturelles, touristiques mais aussi économiques.

Pour bien comprendre que l'antagonisme apparent entre l'urbanité et la naturalité n'en est pas un, il convient de distinguer le processus d'urbanisation d'une part, et les pratiques, les formes d'appropriation de l'espace urbain d'autre part. Ainsi, l'extension de la ville d'un point de vue morphologique n'est pas un prérequis au développement d'un mode de vie urbain ; et, inversement, l'expansion de l'espace bâti et artificialisé n'induit pas nécessairement une plus-value dans la manière d'habiter la ville. Une telle dissociation se retrouve à Stockholm où l'instauration du Parc national urbain a pour finalité d'encadrer l'urbanisation tout en renforçant l'urbanité globale de la métropole. Par exemple, parmi les arguments des acteurs qui défendaient la création du Parc national urbain au début des années 1990, il y a la contestation d'un projet de bouclage du contournement routier du centre de Stockholm qui traverserait - en grande partie en tunnel - 
l'île de Djurgården (carte 1). En 1997, deux ans après l'instauration du Parc national urbain, ce projet d'aménagement d'ampleur fut temporairement écarté, pour être repensé. Aujourd'hui, il est donc toujours promu par certains acteurs économiques et institutionnels comme un projet d'avenir pour les prochaines décennies, notamment par la ville de Stockholm ${ }^{17}$, alors que d'autres s'y opposent. Les premiers se placent dans une logique d'équipement de l'espace alors que les seconds prônent plutôt une stratégie de ménagement. Cette conflictualité environnementale n'est pas spécifique à Stockholm et de nombreux autres exemples de ce genre existent ${ }^{18}$. Dans le cas présent, la tension se joue donc sur des interprétations divergentes de l'intérêt national : ce peut être la transformation d'un espace en bouclant le périphérique de Stockholm afin d'induire des effets positifs sur le développement métropolitain ; ce peut a contrario être la préservation d'un espace en l'état en considérant que toute modification amoindrirait son intérêt social et sa plus-value urbaine. Par ailleurs, des chercheurs ont montré que la création du Parc national urbain a effectivement limité l'urbanisation en son sein, mais qu'elle a accru la pression foncière à ses abords dans une logique de report de l'urbanisation en périphérie du parc $^{19}$.

En tant que vecteur d'identité métropolitaine et en tant qu'espace public, Djurgården apparaît comme une composante essentielle de l'urbanité de Stockholm. Dès lors, le Parc national urbain n'est pas seulement un cadre réglementaire. Il est l'expression d'une protection singulière et, résultant d'un compromis politique sur la conception d'une naturalité urbaine, il peut être envisagé comme la manifestation d'une utopie métropolitaine.

\section{RÉALITÉS ET IMAGINAIRES D’UNE NATURALITÉ URBAINE : LA NATURBANITÉ COMME FIGURE DE L'UTOPIE}

En abordant ce Parc national urbain au prisme de l'utopie, il semble donc pertinent d'approfondir la réflexion sur l'articulation entre l'urbanité et la naturalité suggérée par cet objet spatial. Convoquer l'idée de naturbanité permet à la fois de dire l'imbrication de ces deux formes de rapport au monde et d'envisager d'autres dialectiques, entre pragmatisme et utopisme, entre idéologie et utopie.

\section{Produire la naturalité nordique par l'intégration urbaine}

Au-delà du renversement de la logique conservatrice, une autre facette de l'utopie à laquelle renvoie le Parc national urbain de Stockholm est sa capacité à

17 Stockholms stad, Vision 2030, 2012.

18 Lionel Laslaz, "Conflit environnemental ", Hypergéo, 2015, [http://www.hypergeo.eu/spip. php?article635] (consulté le 27 février 2017).

19 Ylva Uggla, "The Urban Park as "Paradise Contrived” ", in Green Utopianism. Perspectives, Politics and Micro-Practices, K. Bradley et J. Hedrén (éd.), Londres, Routledge, 2014b, p. 150-164. 


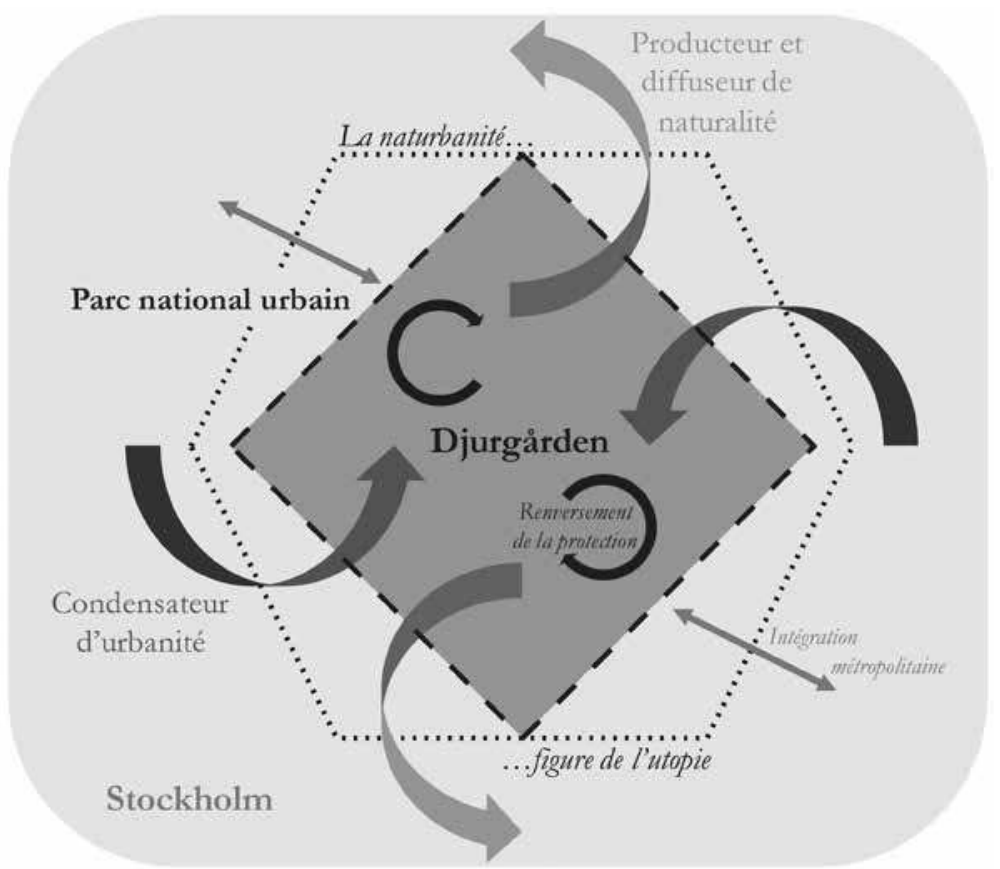

Figure 1 : Le Parc national urbain, une utopie métropolitaine ? Essai de modélisation de la naturbanité.

Réalisation Girault, 2016

façonner la naturalité tout en renforçant l'intégration urbaine de cet espace. Ce paradoxe apparent peut se comprendre aisément. D'abord, cette intégration n'est pas seulement la localisation intra-urbaine de ce parc, elle repose sur «le renforcement des liens qui unissent les éléments d'un système ${ }^{20}$, en l'occurrence sur la capacité de cet espace à faire s'articuler des réseaux et des mobilités, des aménagements et des pratiques, des paysages et des représentations, et, finalement, des réalités et des imaginaires.

À travers ses monuments, ses équipements et sa diversité fonctionnelle, il est possible de représenter les logiques relationnelles d'intégration de ce lieu au reste de l'espace métropolitain (carte 1 ; figure 1). Que ce soit par la quotidienneté ou par des formes de projection, le Parc national urbain concentre une certaine urbanité et diffuse sa naturalité : c'est ce double processus qui donne sens à la naturbanité. Cette idée va au-delà d'une juxtaposition spatiale et elle exprime la cospatialité du Parc national urbain et de l'espace métropolitain, ainsi que la coprésence des modes d'habiter des individus.

20 Bernard Bret, "Les notions d'intégration et de fragmentation, approche géographique ", $B A G F$, 82, 4, 2005, p. 388 . 
Alors que les pays nordiques se caractérisent notamment par de faibles densités, une répartition de la population dissymétrique et de grandes étendues, le Parc national urbain de Stockholm permet le contact entre un lieu de peuplement majeur en Europe du Nord et un espace de naturalité emblématique de la culture suédoise et nordique. La naturalité n'est pas ici à comprendre comme l'absence ou la minimisation de l'intervention humaine sur un milieu; elle ne correspond pas à l'état d'une nature spontanée et antagoniste avec l'artificialité comme l'envisagent les naturalistes et comme on cherche à la conserver dans les parcs nationaux suédois ${ }^{21}$. Dans cette réflexion, la naturalité est entendue dans un paradigme de dépassement des dualismes entre le naturel et l'artificiel, entre l'homme et la nature pour être considérée comme un rapport social, culturel et politique au monde biophysique ${ }^{22}$. En ce sens, elle diffère également du concept de wilderness dont elle est une piètre traduction ${ }^{23}$. Effectivement, ce dernier correspondant plutôt à un degré de naturalité, celui du sauvage. Partant, la naturalité est au même titre que l'urbanité une vision du monde. D'ailleurs, elle est convoquée par certains travaux d'écologie urbaine qui intègrent des savoirs écologiques aux réflexions urbanistiques ${ }^{24}$, et en particulier à propos du Parc national urbain de Stockholm ${ }^{25}$. Aucun antagonisme donc à considérer de concert l'urbanité, ce rapport au monde caractéristique d'un mode d'habiter urbain, et la naturalité. D'ailleurs, plusieurs géographes ont largement exploré cette relation, en considérant que le parc est une figure majeure de l'urbanité et que la nature est une notion plurielle et construite au prisme des urbanités ${ }^{26}$. En somme, le cas du Parc national urbain de Stockholm démontre aussi avec force que la naturalité est une production humaine et, en grande partie, urbaine.

La trajectoire aboutissant à l'affirmation d'un haut lieu de la naturalité nordique au centre de Stockholm est particulièrement évocatrice de ce processus. En effet, alors qu'elles constituaient des espaces agricoles propriétés de l'Église et situées en périphérie immédiate du centre historique de Stockholm, les terres de Djurgården deviennent royales au début du XVI e siècle, à la faveur d'une décision du roi de Suède Gustave Ir Vasa. C'est un des moyens et des résultats concrets de

21 Claes Grundsten, National Parks in Sweden. Europe's Last Wilderness, Stockholm, National Environmental Protection Board, 1987.

22 Philippe Descola, Par-delà nature et culture, Paris, Gallimard, 2005 ; Serge Moscovici, Essai sur l'histoire humaine de la nature, Paris, Flammarion, 1968.

23 Paul Arnould, Éric Glon, "Wilderness, usages et perceptions de la nature en Amérique du Nord », Annales de géographie, 115, 649, 2006, p. 227-238.

24 Jari Niemelä, «Ecology and Urban Planning », Biodiversity and Conservation, 8, 1999, p. 119-131.

25 Thomas Elmqvist, Johan Colding, Stephan Barthel et al., "The Dynamics of Social-Ecological Systems in Urban Landscapes : Stockholm and the National Urban Park, Sweden ", Annals of the New York Academy of Sciences, 1023, 2004, p. 308-322.

26 Augustin Berque, "Des toits, des étoiles », Les Annales de la recherche urbaine, 74, 1, 1997, p. 5-11; Christian Calenge, "Du parc au parc, invocation d'une figure de l'urbanité ", Cahiers de la Méditerranée, 60, 1, 2000, p. 179-201. 


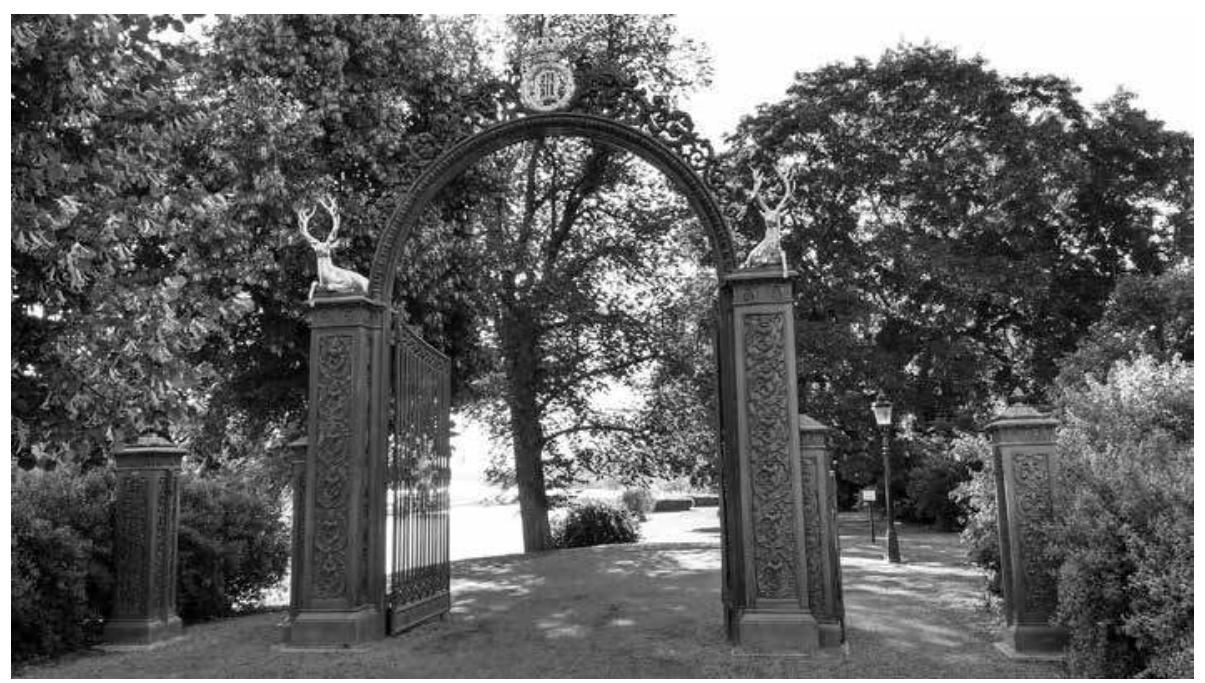

Photographie 3 : Blå Porten, marqueur paysager d'un parc royal fermé devenu Parc national urbain ouvert.

Girault, juillet 2016

l'affirmation du pouvoir royal suédois à cette époque. Suite à ce changement foncier majeur, l'usage cynégétique se développe peu à peu, des parcs animaliers, comprenant notamment des rennes et des cerfs, sont créés - ce qui renvoie d'ailleurs à l'étymologie de "Djurgården " - et, à la fin du XVII e siècle, le roi Charles XI clôture cet espace pour en faire un parc de chasse réservé à la Cour. Un des héritages de cette période est la Porte Bleue (Blå Porten ; photographie 3) qui rappelle aux visiteurs actuels qu'avant d'être un espace public librement accessible, Djurgården a longtemps été un espace réservé à une élite sociale venant pratiquer ses loisirs mondains à proximité des palais royaux, dans des forêts et des prairies entretenues et préservées à cette fin. La naturalité qui fait aujourd'hui l'objet de mesures de protection a donc d'abord été pensée et créée comme une aménité royale avant d'être considérée, depuis le XIX ${ }^{\mathrm{e}}$ siècle, comme un espace de détente et de récréation pour tous les citadins ${ }^{27}$.

La manière dont les habitants se sont approprié ces jardins royaux, par exemple à proximité des trois palais que comporte le parc (photographie 4 ; carte 1), illustre l'importance qu'ils accordent à ce vaste espace ouvert et boisé et, en retour, la manière dont la naturalité de Djurgården est socialement attendue, construite et validée au sein de la métropole suédoise. La production d'un récit national à son endroit et l'évocation d'un lieu paradisiaque à son égard ${ }^{28}$ renforcent

27 Peter Schantz, "The National Urban Park in Greater Stockholm : Background, Legislation and Implementation ", Garden History, 32, 2, 2004, , p. 279-280.

28 Ylva Uggla, 2014 a et b, op. cit. 


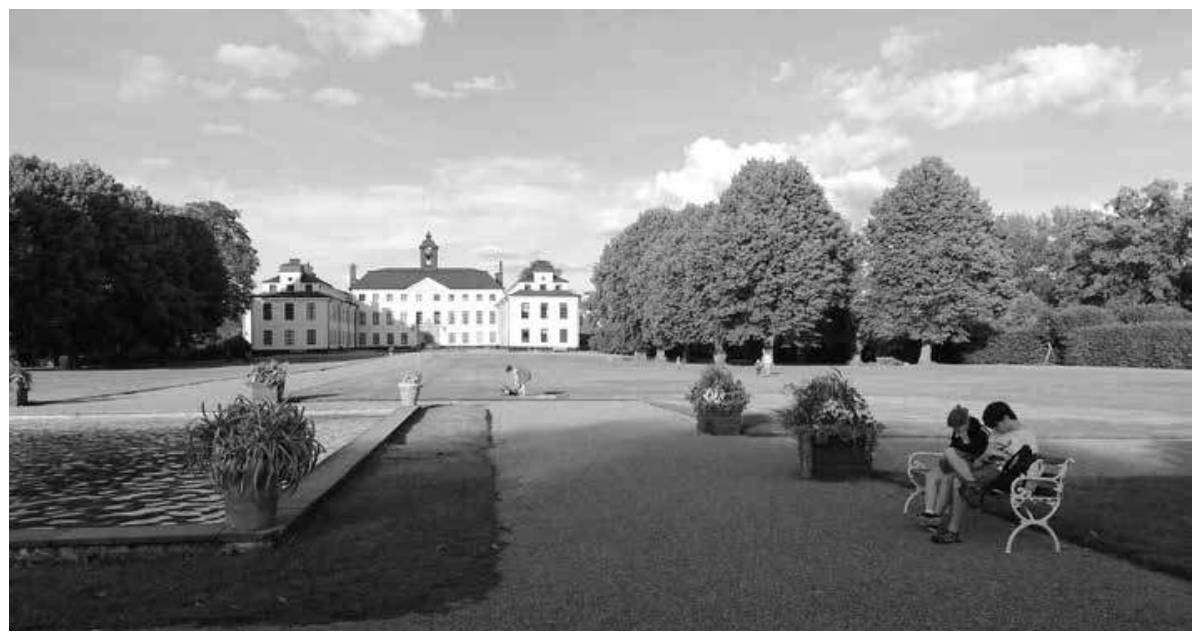

Photographie 4 : Le château d'Ulriksdal au sein du Parc national urbain : un espace public parmi d'autres pour les Stockholmois. Girault, juillet 2016

l'exceptionnalité du lieu et justifient sa protection. Pour autant, il demeure un espace de la quotidienneté dans la vie des citadins et, en ce sens, l'adjectif urbain associé à l'image du parc national révèle toutes ses acceptions. Il n'est plus seulement un parc ayant une haute valeur écologique comme son appellation de parc écologique (Ekoparken) le suggérait au début des années 1990 ; il rassemble des valeurs très diverses (historiques et culturelles, sociales et politiques, environnementales et symboliques) qui nourrissent directement l'urbanité.

\section{Au-delà des oxymores, une utopie ou une idéologie urbaine?}

En prenant un peu de hauteur au sens propre du terme, par exemple en contemplant la partie sud de Djurgården depuis la tour de transmission radio et télévisuelle (photographie 5b ; carte 1), l'observateur se trouve dans une situation originale : le végétal domine largement au premier plan, alors qu'on est au centre de la métropole. À l'exception des quelques monuments emblématiques de cet espace, le bâti semble être à distance. Pourtant cette mise à distance ne signifie pas une mise à l'écart de la ville de Stockholm mais, bien au contraire, la capacité de cette ville à faire de ce vaste espace végétalisé un élément majeur de son urbanité. À l'inverse, vue du sol, la présence de cette grande tour en béton peut également surprendre le visiteur (photographies 5 a et c). En apparaissant aujourd'hui comme un élément patrimonial au même titre que les demeures royales ou les chênes pluricentenaires, elle invite surtout à considérer le développement urbain de Stockholm sur le temps long. L'emprise spatiale de cet espace vert urbain est donc un facteur explicatif parmi d'autres de l'acuité d'une naturalité 

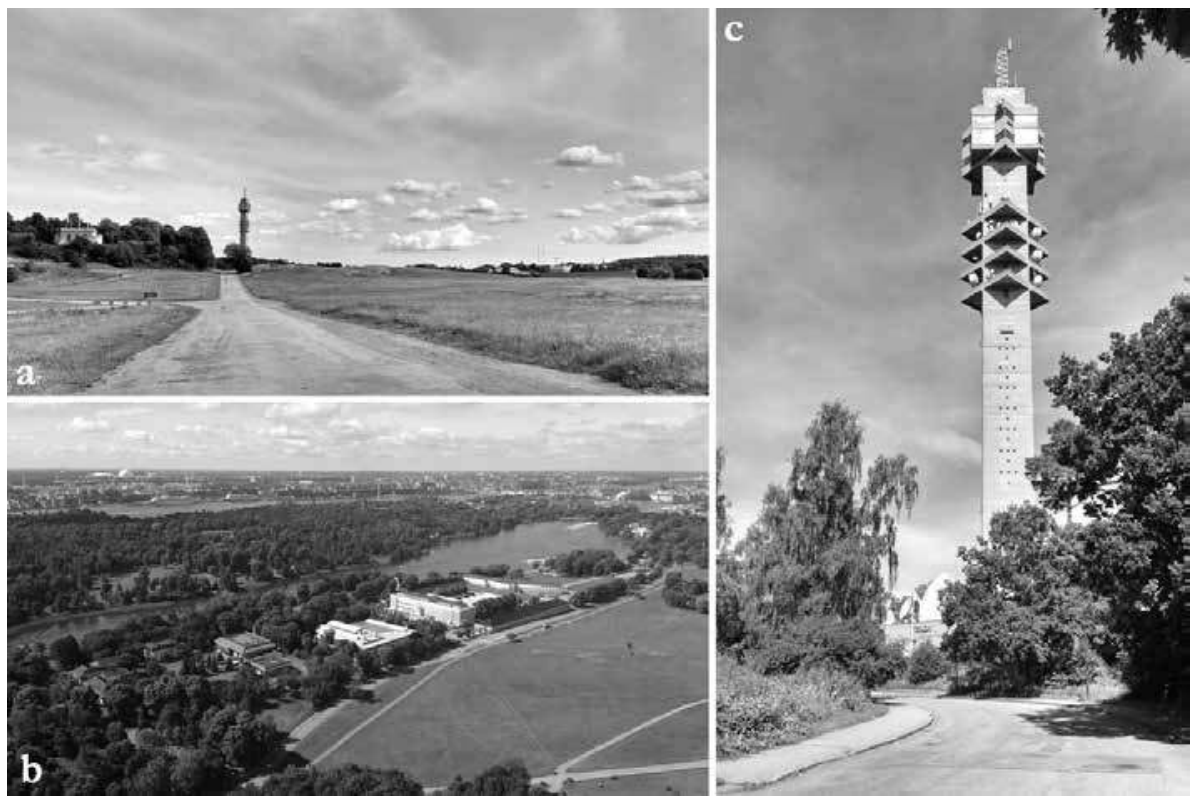

Photographies 5 : La partie sud de l'île de Djurgården et du Parc national urbain de Stockholm vue depuis ou aux abords de la tour des télévisions et radios (Kaknästornet) : plus qu'un espace vert urbain, l'expression d'une naturbanité.

urbaine à la fois réelle et imaginée ${ }^{29}$, mais il n'est pas le seul. La place qu'occupe Djurgården dans l'imaginaire comme dans la quotidienneté des habitants, son rôle dans l'histoire du développement urbain, dans le plan d'urbanisme actuel ${ }^{30}$ comme dans la prospective urbanistique ont contribué à faire de Stockholm une des villes les plus vertes d'Europe et du monde ${ }^{31}$. En effet, cet espace est un élément majeur de la structure écologique de Stockholm et il apparaît comme un nœud central des trames vertes et bleues de cette ville en archipe ${ }^{32}$. Par ailleurs, cette volonté d'affirmer son exemplarité environnementale relève à de nombreux égards d'une stratégie de métropolisation où Stockholm apparaît comme un modèle urbain à la fois pour ce qu'elle est et pour ce que les différents acteurs projettent qu'elle soit ${ }^{33}$.

29 À titre de comparaison, par rapport aux 2700 hectares du Parc national urbain de Stockholm, Central Park (New York) a une superficie de 341 hectares et le parc de la Tête d'Or (Lyon) de 117 hectares.

30 Stockholms stad, The Walkable City. Stockholm City Plan, 2010.

31 Jonathan Metzger, Amy Rader Olsson (dir.), Sustainable Stockholm. Exploring Urban Sustainability in Europe's Greenest City, Londres, Routledge, 2013.

32 Stephan Barthel et al., op. cit.

33 Camille Girault, "L'affirmation de l'exemplarité environnementale comme stratégie de métropolisation des villes nordiques ", EchoGéo, 36, 2016, [http://echogeo.revues.org/14574] (consulté le 27 février 2017). 

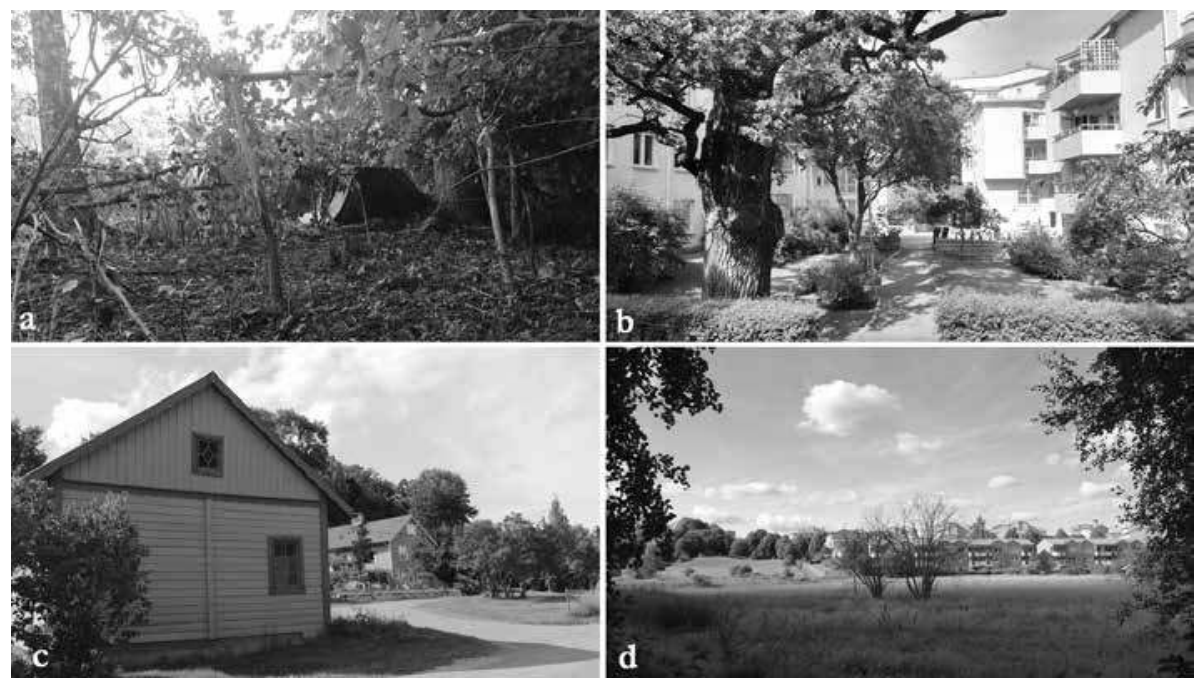

Photographies 6 : Du précaire et informel au résidentiel huppé : expression de la diversité urbaine au sein du Parc national urbain de Stockholm. Girault, juillet 2016

Pour autant, plus de vingt ans après sa création, le Parc national urbain souffre toujours d'un déficit d'appropriation de la part des Stockholmois qui n'ont que partiellement assimilé ce changement de statut. La discrétion du Parc national urbain dans le paysage en tant qu'entité constituée, notamment par la signalétique, en est une preuve (photographies 1a et c). Une double interprétation peut alors être faite : cette discrétion peut soit laisser entendre qu'il s'agit d'un projet politique restant en grande partie à l'état de chimère, soit elle exprime justement la réalité spécifique de cet objet hybride.

De plus, en dépit des apparences d'une gestion sans écueil reposant sur un consensus social d'appropriation d'un lieu paradisiaque, cet objet spatial utopique est rattrapé par la réalité urbaine, notamment par sa diversité. En s'écartant des chemins bien entretenus, une observation attentive des sous-bois montre des phénomènes d'injustice spatiale : pour certains habitants précaires de la ville, le Parc national urbain est d'abord un lieu de refuge où il est possible de survivre à l'abri des regards, à quelques pas de l'hypercentre de Stockholm. Comme ailleurs, cet espace n'est donc pas exempt de formes de relégation sociospatiales. Ainsi, nous avons pu relever quelques campements de fortune se localisant dans des sous-bois de Djurgården (photographie 6a ; carte 1). Ici l'ampleur de l'installation d'une population de sans domicile fixe est bien moindre que celle observée par exemple au bois de Vincennes à Paris ${ }^{34}$, mais elle vient rappeler qu'en dépit de l'image

34 Étienne Grésillon, Jean-Paul Amat, Aurélie Tibaut, «Les "sans domicile fixe” du bois de Vincennes : une précarité dans des espaces de durabilité ", Géocarrefour, 89, 2014, p. 261-269. 
idéalisée qu'elles ont souvent à l'étranger, les sociétés nordiques produisent aussi des inégalités. D'ailleurs, à quelques pas de ces formes d'habitat précaire et temporaire, certains logements suggèrent au contraire que pour d'autres habitants de Stockholm, Djurgården est un lieu de résidence privilégié permettant l'idéal d'une vie à la campagne dans la ville (photographie 6c). Ces inégalités manifestes, qui plus est dans une société nordique au niveau de vie moyen élevé et aux inégalités moindres ${ }^{35}$, viennent écorner l'image et le récit d'un lieu qui serait l'expression de la liberté, de l'équité et de la justice environnementale.

En allant plus avant dans l'analyse, un tel idéal qui se révèle en réalité socialement sélectif tend à figer, voire à exacerber, une situation spatiale de justice sociale insatisfaisante. Alors que le statut de Parc national urbain affirme que Djurgården est un bien public accessible à tous, les pratiques observées semblent confirmer qu'il préserve les valeurs paysagères, patrimoniales et écosystémiques mais qu'il maintient aussi une certaine inégalité sociale d'accès et de jouissance à ces aménités. Ainsi, en s'appuyant sur les éclairages philosophiques de Paul Ricœur, considérer que le Parc national urbain est une utopie serait constater qu'il manifeste une alternative à la réalité présente par une projection des possibles. Pourtant les observations conduites dans cet espace et les réflexions à son égard montrent qu'il est plutôt une légitimation d'une réalité, reflétant de ce fait davantage une idéologie ${ }^{36}$. L'hypothèse de l'hétérotopie semble donc à privilégier.

\section{Conclusion}

Le Parc national urbain, tel qu'il a été conçu et mis en œuvre à Stockholm, apparaît d'abord comme un objet spatial singulier, original et novateur. Il n'est pas un simple espace vert urbain, il n'est pas non plus un espace protégé à l'instar des réserves naturelles ou des parcs nationaux ; il résulte plutôt d'objectifs variés, innovants et pour partie contradictoires qui en font un objet hybride. Dans sa manière de condenser l'urbanité et de produire la naturalité, il mobilise le registre de l'innovation, de l'idéalisation, de l'invention et renvoie ainsi à la figure de l'utopie. D'un autre côté, dans sa façon de préserver un état urbain établi, de patrimonialiser un territoire et d'affirmer une identité métropolitaine stabilisée, il exprime plus une idéologie qu'un idéal.

L'utopie existerait donc, mais de façon partielle. Elle semble réalisée en matière de naturbanité, mais elle reste à réaliser en termes de justice environnementale et d'égale accessibilité. À l'aune de la mise en œuvre du Parc national urbain, elle se dessinerait et s'affirmerait par une hybridité spatiale intégrant une grande diversité (sociale, patrimoniale, écosystémique) et une certaine densité (de pratiques,

35 Yohann Aucante, Les démocraties scandinaves. Des systèmes politiques exceptionnels ?, Paris, A. Colin, 2013.

36 Paul Ricœur, L'idéologie et l'utopie, Paris, Seuil, 1997. 
de représentations, de symboles). Cette conception utopique est donc d'abord l'ambition d'un écart aboli, en raison de choix politiques, entre les citadins et la dimension naturelle de leur ville. L'utopie ne serait donc que superficielle, elle n'aurait de sens que depuis l'extérieur alors qu'en pratique et au quotidien le Parc national urbain de Stockholm est un espace urbain fonctionnel et relationnel aux prises avec le réel. On peut y voir l'expression d'une naturbanité qui, au-delà du néologisme, réfere à une certaine réalité et une certaine idée de la ville. Un retour critique aux fondements de l'utopie permet d'envisager cet objet spatial comme une innovation métropolitaine qui remet en cause les modalités des politiques de protection tout en les utilisant pour consolider l'identité de la capitale suédoise.

En appui de cette réflexion, un essai de modélisation est proposé pour visualiser de manière synthétique les différents processus à l'œuvre et les diverses pistes analytiques qu'il est possible d'emprunter à partir d'une observation du Parc national urbain de Stockholm (figure 1). Les flèches (directement légendées sur la figure) montrent le caractère dynamique, construit et systémique de la naturbanité entendue comme utopie métropolitaine. 